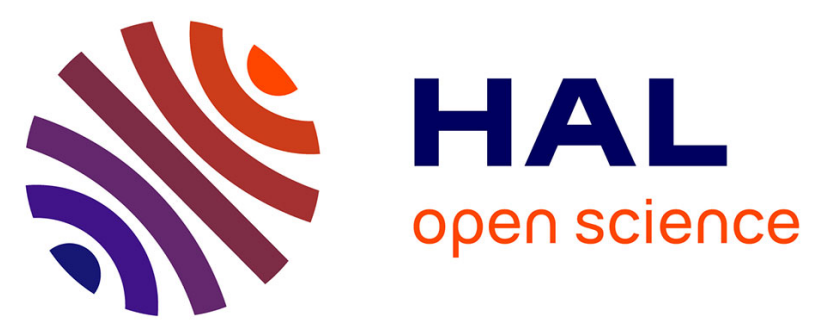

\title{
A shared control solution for safe assisted power wheelchair navigation in an environment consisting of negative obstacles: a proof of concept
}

Louise Devigne, François Pasteau, Tom Carlson, Marie Babel

\section{To cite this version:}

Louise Devigne, François Pasteau, Tom Carlson, Marie Babel. A shared control solution for safe assisted power wheelchair navigation in an environment consisting of negative obstacles: a proof of concept. SMC 2019 - IEEE International Conference on Systems, Man, and Cybernetics, Oct 2019, Bari, Italy. pp.1043-1048, 10.1109/SMC.2019.8914211 · hal-02263532

HAL Id: hal-02263532

https://hal.inria.fr/hal-02263532

Submitted on 5 Aug 2019

HAL is a multi-disciplinary open access archive for the deposit and dissemination of scientific research documents, whether they are published or not. The documents may come from teaching and research institutions in France or abroad, or from public or private research centers.
L'archive ouverte pluridisciplinaire HAL, est destinée au dépôt et à la diffusion de documents scientifiques de niveau recherche, publiés ou non, émanant des établissements d'enseignement et de recherche français ou étrangers, des laboratoires publics ou privés. 


\title{
A shared control solution for safe assisted power wheelchair navigation in an environment consisting of negative obstacles: a proof of concept
}

\author{
Louise Devigne $^{1}$, François Pasteau ${ }^{1}$, \\ Tom Carlson ${ }^{2}$, Marie Babel ${ }^{1}$
}

\begin{abstract}
Power wheelchairs allow people with motor disabilities to have more mobility and independence. However, driving safely such a vehicle is a daily challenge particularly in urban environments while navigating on sidewalks, negotiating curbs or dealing with uneven grounds. Indeed, differences of elevation have been reported to be one of the most challenging environmental barrier to negotiate, with tipping and falling being the most common accidents power wheelchair users encounter. It is thus our challenge to design assistive solutions for power wheelchair navigation in order to improve safety while navigating in such environments. To this aim, we propose a shared-control algorithm which provides assistance while navigating with a wheelchair in an environment consisting of negative obstacles. We designed a dedicated sensor-based control law allowing trajectory correction while approaching negative obstacles e.g. steps, curbs, descending slopes. This shared control proposed method takes into account the humanin-the loop factor. In this study, our solution the ability of our system to ensure a safe trajectory while navigating on a sidewalk is demonstrated through simulation, thus providing a proof-of-concept of our method.
\end{abstract}

\section{INTRODUCTION}

Motorized mobility is not without risk and accidents can occur while driving a power wheelchair. In a study on the prevalence of wheelchair-related accidents involving 95 participants, $54.7 \%$ of them reported at least 1 accident in the past 3 years [1]. In this context, robotic assistive solutions can increase wheelchair navigation safety. Indeed, navigation support can be supplied in the form of autonomous [2] [3] or semi-autonomous [4] assistance [5].

However, the existing navigation assistance technologies are currently restricted to indoor environment. Indeed, they do not detect outdoor environmental barriers such as differences in elevation (step, curb, etc.). Yet, recent studies highlight the benefits of power mobility in the case of outdoor activities [6] [7]. Indeed, it is reported that power mobility can improve the user self-esteem and sense of independence as it allows to go outdoors more easily and to have the opportunity to socialize and enjoy daily activities such as shopping. Therefore, outdoor navigation solutions have to be developed to increase navigation safety. Among the 3 categories of wheelchair-related accidents identified in [1], 87.7\% were tips and falls. In some cases, this type of accident can have serious consequences such as traumatic brain injury or spinal cord injury [8]. Moreover, [9] highlights that environmental obstacles impact the outdoor driving safety and that one of

\footnotetext{
${ }^{1}$ Univ Rennes, INSA, CNRS, Inria, Irisa-UMR6074, Rennes, France.

2 Aspire Centre for Rehabilitation Engineering and Assistive Technology, University College London, UK.
}

the biggest challenges is to negotiate curb drop-offs and curb cuts. While a lot of works have been done in the robotics field on detecting and avoiding obstacle, there is significantly less research on detection of negative obstacles to improve outdoor robot navigation. In [10] an algorithm analyzes the terrain using a 3D laser range finder. They classify the obstacles and populate a grid map of obstacles and terrain features. In [11], a stereo-based scene reconstruction is used to detect drop-offs and also relies on the definition of a grid map. The few proposed methods are used for autonomous robot navigation and are typically based on stereo-vision detection or use expensive sensors such as LIDARs and generally rely on computationally expensive localization methods within local maps of the environment.

As for wheelchair navigation, only a few research teams have worked on assistive solutions for power wheelchair driving in outdoor environments. While the intelligent wheelchairs presented in [12] simply stops when a drop-off is detected, [13] proposes an algorithm relying on depth information using stereo vision for finding obstacle edges. Then, if some efforts have been made in order to detect drop-offs using a robotized power wheelchair, no control law is given in order to assist the user while driving. In [14] we presented an approach dealing with obstacles that a wheelchair cannot cross (e.g. walls, furniture).

In this paper, we present a complementary approach providing navigation assistance in an environment consisting of negative obstacles which are defined here as curbs, steps and descending slopes. Here, we use distance sensors mounted all around the wheelchair to detect distances to the floor and to extract local floor orientations information. With this method, no map is required and the proposed solution is able to smoothly correct the trajectory of the wheelchair while approaching a negative obstacle while respecting user intention.

The paper is organized as follows. Section II deals with the modeling related to the robotized wheelchair. Section III presents the computation of the constraints deduced from sensors measurements on which the method relies and then details the proposed sensor-based wheelchair assisted navigation method. Section IV presents simulations of the system, thus highlighting the behavior of the servoing process in presence of negative obstacles while driving on a virtual sidewalk. The results are discussed in section V. 


\section{MODELING}

We model a 6 wheeled robotic wheelchair composed of 2 actuated wheels and 4 passive caster wheels. It is controlled with 2 variables, namely the linear velocity $u$ and the rotational velocity $\omega$.

The wheelchair is equipped with distance sensors. As shown on Fig. 1, we define the following notations

- let $\mathcal{F}_{R}\left(R, \vec{X}_{R}, \overrightarrow{Y_{R}}, \overrightarrow{Z_{R}}\right)$ be the frame attached to the robotic wheelchair,

- let $\mathcal{F}_{s_{i}}\left(S_{i}, \vec{X}_{i}, \vec{Y}_{i}, \vec{Z}_{i}\right)$ be the frame attached to the sensor $s_{i}$,

- let $\mathbf{u}=(v, \omega)$ be the 2-DoF control input of the wheelchair,

- let $\mathbf{v}_{s_{i}}$ be the velocity of the sensor $s_{i}$,

- let $a_{i}$ and $b_{i}$ be the distances from the robot frame to the sensor frame respectively along the robot $x$-axis and $y$-axis.

- let $\phi_{i}$ be the orientation of the sensor $s_{i}$ with respect to the robot frame Oxy plane,

- let $d_{i}$ be the distance measured by the sensor $s_{i}$,

- let $\theta_{i}$ be the orientation of the sensor $s_{i}$ with respect to the robot frame $\mathrm{z}$-axis,

- let $e_{i}$ be the difference between the measured distance $x_{i}$ and its desired value $x_{i}^{*}$.

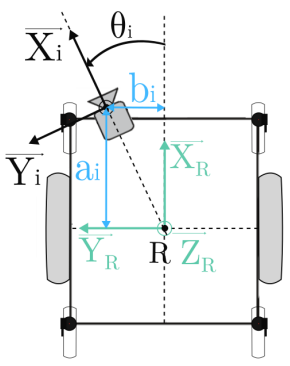

(a) Details for one sensor $s_{i}$ (top view).

Fig. 1: Definition of the robot frame

Each sensor $s_{i}$ is rigidly fixed to the robot frame so that the translation vector ${ }^{r} \mathbf{t}_{s_{i}}$ between $\mathcal{F}_{s_{i}}$ and $\mathcal{F}_{R}$ is such that

$$
{ }^{r} \mathbf{t}_{s_{i}}=\left[\begin{array}{c}
a_{i} \\
b_{i} \\
0
\end{array}\right]
$$

The rotation matrix ${ }^{s_{i}} \mathbf{R}_{r}$ relative to sensor $s_{i}$ frame $\mathcal{F}_{s_{i}}$ with respect to the robot frame $\mathcal{F}_{R}$ is given by

$$
{ }^{s_{i}} \mathbf{R}_{r}={ }^{s_{i}} \mathbf{R}_{r}\left(\phi_{i}\right)^{s_{i}} \mathbf{R}_{r}\left(\theta_{i}\right)
$$

with

$$
{ }^{s_{i}} \mathbf{R}_{r}\left(\phi_{i}\right)=\left[\begin{array}{ccc}
\cos \phi_{i} & 0 & -\sin \phi_{i} \\
0 & 1 & 0 \\
\sin \phi_{i} & 0 & \cos \phi_{i}
\end{array}\right]
$$

and

$$
{ }^{s} \mathbf{R}_{r}\left(\theta_{i}\right)=\left[\begin{array}{ccc}
\cos \theta_{i} & \sin \theta_{i} & 0 \\
-\sin \theta_{i} & \cos \theta_{i} & 0 \\
0 & 0 & 1
\end{array}\right]
$$

The translation ${ }^{s_{i}} \mathbf{t}_{r}$ between the robot $\mathcal{F}_{R}$ frame and the sensor $s_{i}$ frame $\mathcal{F}_{s_{i}}$ is obtained from ${ }^{s_{i}} \mathbf{R}_{r}$ with

$$
{ }^{s_{i}} \mathbf{t}_{r}={ }^{s_{i}} \mathbf{R}_{r}{ }^{r} \mathbf{t}_{s_{i}} .
$$

From ${ }^{s_{i}} \mathbf{R}_{r}$ and ${ }^{s_{i}} \mathbf{t}_{r}$, the velocity screw transformation matrix is given by

$$
{ }^{s_{i}} \mathbf{W}_{r}=\left[\begin{array}{cc}
{ }^{s_{i}} \mathbf{R}_{r} & {\left[{ }^{s_{i}} \mathbf{t}_{r}\right]_{\times}{ }^{s_{i}} \mathbf{R}_{r}} \\
\mathbf{0}_{3 \times 3} & { }^{s_{i}} \mathbf{R}_{r}
\end{array}\right]
$$

with []$_{\times}$the skew matrix expression.

The velocity $\mathbf{v}_{s_{i}}$ of the sensor $s_{i}$ in the sensor frame $\mathcal{F}_{s_{i}}$ is derived from the robot 2-DoF control input $\mathbf{u}$ in the robot frame using

$$
\mathbf{v}_{s_{i}}={ }^{s_{i}} \mathbf{T}_{r} \mathbf{u}
$$

with

$$
{ }^{s_{i}} \mathbf{T}_{r}={ }^{s_{i}} \mathbf{W}_{r}{ }^{r} \mathbf{J}_{r}
$$

where ${ }^{r} \mathbf{J}_{r}$ is the robot jacobian expressed in the robot frame such that

$$
{ }^{r} \mathbf{J}_{r}=\left[\begin{array}{llllll}
1 & 0 & 0 & 0 & 0 & 0 \\
0 & 0 & 0 & 0 & 0 & 1
\end{array}\right]^{T} .
$$

\section{SENSOR-BASED SERVOING FOR ASSISTED NAVIGATION ON A SIDEWALK}

In [14], we presented a semi-autonomous approach providing adaptive assistance in the form of an intuitive obstacle avoidance. This method consists in merging the user control coming from a wheelchair controller with a set of constraints deduced from the sensors. This shared control law is based on the definition of two distinct areas in the wheelchair velocity domain that represent allowed and forbidden wheelchair control input values. So far, we applied this method to indoor navigation. In this section, we present the assisted power wheelchair navigation in an environment consisting of environment obstacles by detailing the features extracted from the sensors measurements and we present the computation of the constraints in the velocity domain. The resulting computation of the wheelchair control values from user velocity input by minimizing a cost function is directly applied from [14].

\section{A. Distance sensor features}

In this method, we want to extract relevant information from each sensor distance measurement. Considering a sensor able to measure multiple distances within different regions of interest, we are able to collect information about floor differences of elevations by means of local floor orientation computation obtained for each sensor. This leads to the definition of two features in the form of angles characterizing the orientation of the detected floor area. As shown on Figure 2 , let $\left(\alpha_{x_{i}}, \alpha_{y_{i}}\right)$ be the set of angles defining the orientation of the floor with respect to the wheelchair $O x y$ plane with $\alpha_{x_{i}}$ and $\alpha_{y_{i}}$ being defined respectively around the sensor $s_{i}$ $x$-axis and $y$-axis. 


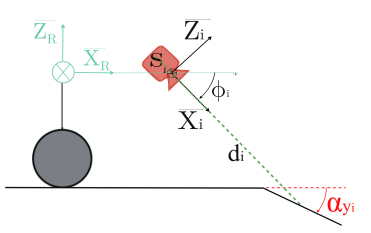

(a) Definition of $\alpha_{y_{i}}$.

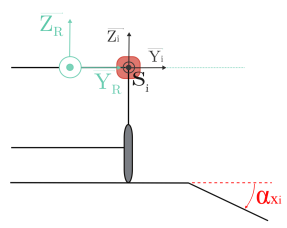

(b) Definition of $\alpha_{x_{i}}$.

Fig. 2: Definition of $\alpha_{x_{i}}$ and $\alpha_{y_{i}}$.

\section{B. Velocity regulation controller design}

Within the context of power wheelchair navigation assistance in an environment consisting of negative obstacles, a velocity controller is designed to ensure the wheelchair to navigate safely. We propose here to progressively adapt the wheelchair control input to avoid hazardous situations caused by negative obstacles.

To do so, we define the error $e_{s_{i}}$ for each sensor $s_{i}$ such that $e_{s_{i}}=d_{i}-d_{i}^{*}$ where $d_{i}, d_{i}^{*} \in \mathbb{R}^{+}$are respectively the distance measured by a sensor $s_{i}$ to the floor and a configurable fixed value corresponding to the maximum allowed distance from the sensor $s_{i}$ to the floor.

1) Interaction matrix computation: With the 2 features $\alpha_{x_{i}}$ and $\alpha_{y_{i}}$ defined in III-A, we can design a dedicated control law that regulates the error $e_{s_{i}}$ by taking into account local floor area orientation into each sensor $s_{i}$ interaction matrix.

For the present case, the interaction matrix $\mathbf{L}_{x_{i}}$ is such that

$\mathbf{L}_{d_{i}}=\left[\begin{array}{llllll}-1 & \frac{\tan \alpha_{x_{i}}}{\sin \phi_{i}} & \frac{1}{\tan \left(\phi_{i}+\alpha_{y_{i}}\right)} & 0 & \frac{d_{i}}{\tan \left(\phi_{i}+\alpha_{y_{i}}\right)} & d_{i} \frac{\tan \left(\alpha_{x_{i}}\right)}{\sin \phi_{i}}\end{array}\right]$,

where $\alpha_{x_{i}}$ and $\alpha_{y_{i}}$ are depicted in Figure 2.

2) Shared control method: The objective is to regulate the wheelchair velocity control input to avoid hazardous situations involving negative obstacles when needed while still allowing full control to the user when there is no negative obstacle close to the wheelchair, we propose to gradually activate the regulation of each distance sensor $s_{i}$ feature $d_{i}$ to a desired value $d_{i}^{*}$ corresponding to the distance measured when no negative obstacle is detected. This is achieved by using an interval $\left[d_{i}^{s-}, d_{i}^{s+}\right]$ that we define to trigger the sensor-based servoing when the distance $d_{i}$ leaves the safe interval. This hybrid control law method has been introduced in [15] and applied to wheelchair navigation in [16] within a wall avoidance task. The idea is to adapt this framework to our semi-autonomous navigation task in an environment consisting of negative obstacles. We propose the following sensor-based control law aiming to keep the distance feature $d_{i}$ within the interval $\left[d_{i}^{s-}, d_{i}^{s+}\right]$

$$
\dot{d}_{i}=h_{d_{i}} \mathbf{L}_{d_{i}} \mathbf{v}_{s_{i}}
$$

with $\mathbf{L}_{d_{i}} \in \mathbb{R}^{1 \times 6}$ the interaction matrix introduced in [17] and $h_{d_{i}} \in \mathbb{R}^{+*}$ a varying weight associated to the distance feature $d_{i}$. The weight $h_{d_{i}}$ associated to $d_{i}$ is such that $h_{d_{i}} \in$ $[0 ; 1]$. A null weight leads to no regulation of the error $e_{i}=$ $d_{i}-d_{i}^{*}$ by the sensor-based servoing. Therefore, the term $h_{d_{i}}$ allows to activate or deactivate the control law when desired. In order to gradually activate the control law when the feature $d_{i}$ is leaving the safe interval $\left[d_{i}^{s-}, d_{i}^{s+}\right]$, we propose here to define the weight $h_{d_{i}}$ by the following smooth function

$h_{d_{i}}\left(d_{i}\right)=\left\{\begin{array}{cc}\left(1-\cos \left(\pi \frac{d_{i}-d_{i}^{s+}}{d_{i}^{+}-d_{i}^{s+}}\right)\right) / 2 & \text { if } d_{i}^{s+}<x<d_{i}^{+} \\ 0 & \text { if } d_{i}^{s-}<x<d_{i}^{s+} \\ \left(1-\cos \left(\pi \frac{d_{i}-d_{i}^{s-}}{d_{i}^{-}-d_{i}^{s-}}\right)\right) / 2 & \text { if } d_{i}^{-}<x<d_{i}^{s-} \\ 1 & \text { otherwise }\end{array}\right.$

where $\left[d_{i}^{-}, d_{i}^{+}\right] \supset\left[d_{i}^{s-}, d_{i}^{s+}\right]$ is a tolerated interval whose fixed limits represent the distance values corresponding to distances measured to obstacles the wheelchair should avoid. The evolution of the weighting term $h_{d_{i}}$ is given in Fig. 3 .

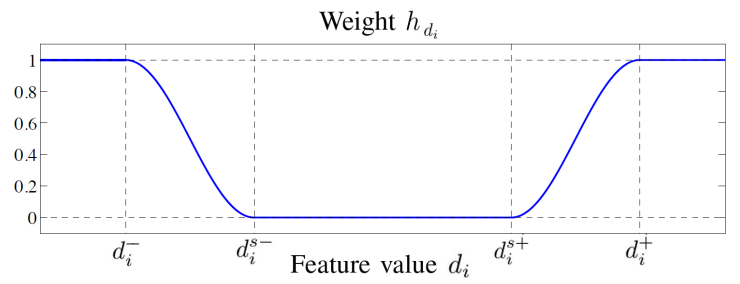

Fig. 3: Weighting function $h_{d_{i}}$ defined for feature $d_{i}$. The weight is null in the safe interval and smoothly increases up to 1 in the tolerated intervals $\left[d_{i}^{-}, d_{i}^{s-}\right]$ and $\left[d_{i}^{s+}, d_{i}^{+}\right]$.

For each sensor $s_{i}$, we have the formulation

$$
\dot{d}_{i} \geq-\lambda e_{s_{i}}
$$

where $\lambda \in \mathbb{R}^{+*}$ is a gain and $e_{s_{i}}=d_{i}-d_{i}^{*}$ the difference between the measured distance to the floor $d_{i}$ and its desired value $d_{i}^{*}$. In this framework, $d_{i}^{*}$ value is the distance which is measured by the sensor $s_{i}$ while detecting no obstacle.

This way, we constrain $\dot{d}_{i}$ by a minimum value $-\lambda e_{s_{i}}$. When considering the relationship $\dot{d}_{i}=\mathbf{L}_{d_{i}}{ }^{s_{i}} \mathbf{T}_{r} \mathbf{u}$ between the 2 DoF wheelchair control input $\mathbf{u}$ and the variation $\dot{d}_{i}$ of the distance $d_{i}$ measured by the sensor $s_{i}$, we obtain

$$
h_{d_{i}} \mathbf{L}_{d_{i}}{ }^{s_{i}} \mathbf{T}_{r} \mathbf{u} \geq-\lambda e_{s_{i}}
$$

i.e.

$$
\mathbf{J}_{s_{i}} \mathbf{u} \geq-\lambda e_{s_{i}}
$$

where $\mathbf{J}_{s_{i}}=h_{d_{i}} \mathbf{L}_{d_{i}}{ }^{s_{i}} \mathbf{T}_{r}$ is the sensor $s_{i}$ jacobian.

The system (15) is a linear equation system which can admit an infinity of solutions in the form of a 2D half-plane in the wheelchair velocity domain.

Defining $\mathbf{A}_{i}=\mathbf{J}_{s_{i}}$ and $B_{i}=-\lambda e_{s_{i}}$, inequation (15) can also be written as

$$
\mathbf{A}_{i} \mathbf{u} \geq B_{i}
$$

This defines 2 half-planes in the $(u, \omega)$ such as shown on Fig. 4a. The green and red half planes respectively represent allowed and forbidden wheelchair control input values. 
In the particular case where $h_{d_{i}}$ is null (i.e. when no negative obstacle is detected), the forbidden half plane is not defined, therefore the constraint deduced from sensor $s_{i}$ is not involved in the regulation of the wheelchair control input. This way, user full control is ensured.

When considering $\mathrm{N}$ sensors, we rewrite (16) as

$$
\mathbf{A} \mathbf{u} \geq \mathbf{B}
$$

using $\mathbf{A}=\left[\begin{array}{c}\mathbf{A}_{0} \\ \vdots \\ \mathbf{A}_{N-1}\end{array}\right]$ and $\mathbf{B}=\left[\begin{array}{c}B_{0} \\ \vdots \\ B_{N-1}\end{array}\right]$. which defines a convex polygon in the wheelchair velocity domain as shown on Fig.4b.

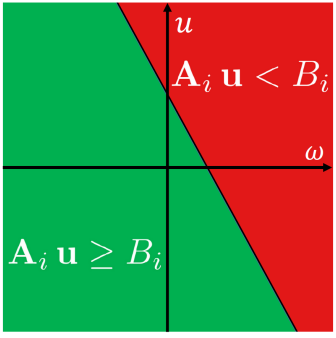

(a) Half plane in the velocity (b) domain.

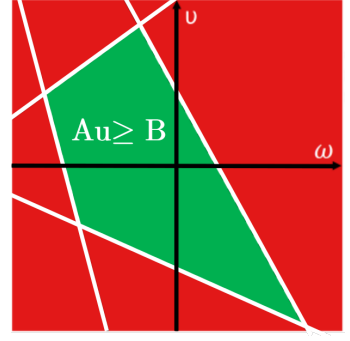

(b) Convex polygon in the wheelchair velocity domain
Fig. 4: Wheelchair setup for the simulation.

\section{Computing control values}

- Let $\mathbf{u}_{o p}=\left(u_{o p}, \omega_{o p}\right)$ be the user desired velocity,

- let $\mathbf{u}_{c m d}=\left(u_{c m d}, \omega_{c m d}\right)$ be the robot velocity.

$\mathbf{u}_{c m d}$ is computed from $\mathbf{u}_{o p}$ under constraints (17) by minimizing a cost function $f$. This can be written as an optimization problem using

$$
\left\{\begin{aligned}
\mathbf{u}_{c m d} & =\min _{\mathbf{u}} f\left(\mathbf{u}_{o p}, \mathbf{u}\right) \\
\mathbf{A} \mathbf{u} & \geq \mathbf{B}
\end{aligned}\right.
$$

The details of the computation of the wheelchair control values from user velocity input by minimizing the function $f$ are given in [14].

\section{Simulations}

In this section, we present the results of a simulation we performed as a first step to develop the proposed solution of power wheelchair navigation assistance on a sidewalk.

\section{A. Simulation framework}

As a part of our research activities, we developed a simulator for assisted power wheelchair navigation. This simulator has been designed with Unity3D and consists of a Virtual Environment in which we operate a virtual robotized wheelchair equipped with virtual distance sensors [18]. In this study, we use a framework designed to control a wheelchair in a virtual environment with any type of assistance (Fig. 5). This framework allows us to develop, test and tune driving assistance solutions more rapidly than while working with a real ground prototype. With such a simulator, we are able to modify the environment and the tests conditions with rapidity and flexibility.

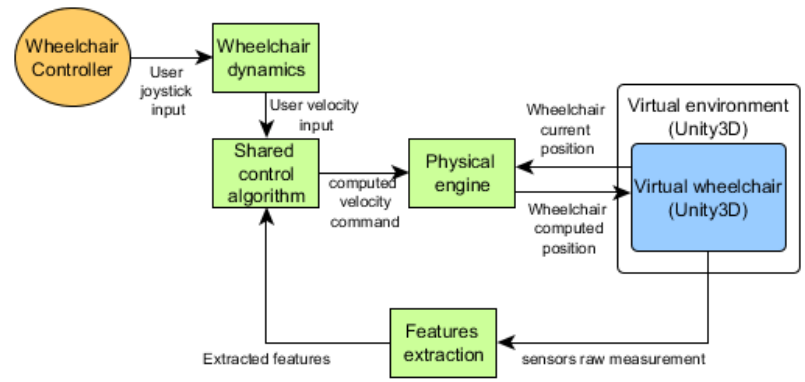

Fig. 5: Framework used for this study on the wheelchair driving simulator.

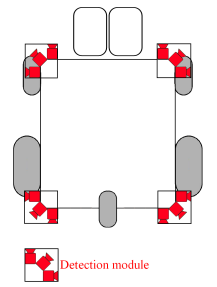

(a) Sensor arrangement.

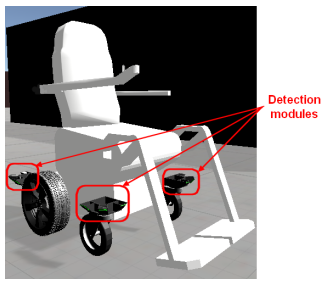

(b) Virtual wheelchair.
Fig. 6: Wheelchair setup for the simulation.

The virtual wheelchair (Fig. 6b) is here equipped with 12 distance sensors divided into 4 detection modules placed at each corner of the virtual wheelchair (Fig. 6a). The virtual environment is displayed on a computer screen as it is only used to demonstrate the properties of the proposed solution for assisted power wheelchair navigation on a sidewalk. As shown on Fig. 7, it consists of a $20 \mathrm{~cm}$ height sidewalk. The velocity parameters of the wheelchair are chosen to mimic a typical commercially-available wheelchair, such that the maximum forward speed is $0.7 \mathrm{~m} . \mathrm{s}^{-1}$ and the maximum rotational speed is 0.5 rad.s ${ }^{-1}$.
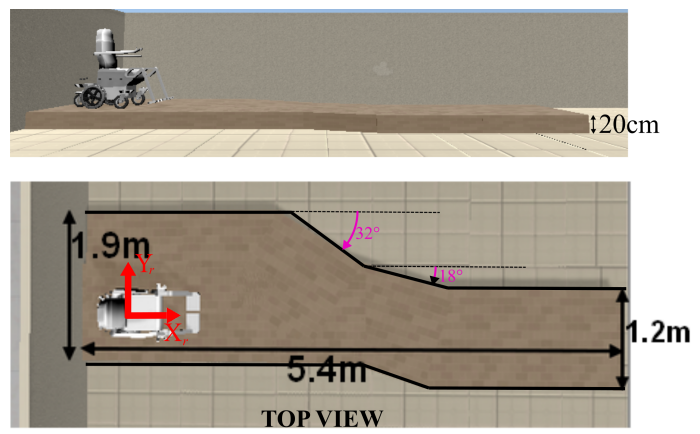

Fig. 7: Virtual environment for the simulation.

\section{B. Simulation results}

Multiple simulation tests have been performed with different initial wheelchair positions. Fig. 8 shows the wheelchair 
trajectories for 3 different initial positions. In order to demonstrate the behavior of the wheelchair and evolution of wheelchair control inputs, we present the results of a single trial on Fig. 9. The user's linear and angular inputs are constant and respectively set to the maximum linear velocity value and zero for angular velocity. This leads the wheelchair to go forward at maximum speed.
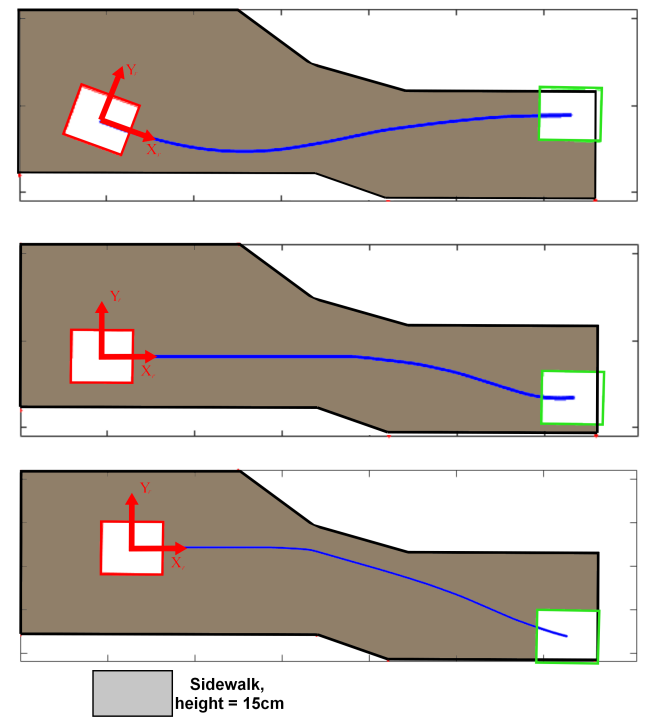

Fig. 8: Wheelchair corrected trajectory for different initial positions.

At the beginning, no negative obstacle is detected: the wheelchair is going towards the curb. Then, when the curb is detected, the linear velocity progressively decreases and the rotational velocity is modified in order to follow the curb. When the wheelchair drives parallel to the curb, the linear velocity increases as there is no danger to fall from the curb. Then, when the wheelchair is driving close to the curb dropoff, an adaptation of both velocities is performed in order to provide a curb-following behavior. When the wheelchair arrives at the end of the corridor in front of a drop-off, it stops and prevents the fall.

\section{DISCUSSION}

The purpose of this study was to provide a proof-ofconcept of a sensor-based shared control method providing navigation assistance in an environment consisting of negative obstacles. The resulting sensor-based driving assistance solution uses 12 low-cost distance sensors detecting distances to the floor around the wheelchair. Considering the sensors being able to detect multiple distances within different regions of interest local floor orientations could thus be determined around the wheelchair. Therefore, each constraint deduced from a sensor measurement is used to define 2 half-planes in the wheelchair velocity domain representing respectively allowed and a forbidden set of values induced by the sensor constraint. Wheelchair control is then computed from user input and sensors constraints so that it respects as much as possible user intention given by $u_{o p}$. The proposed (a)

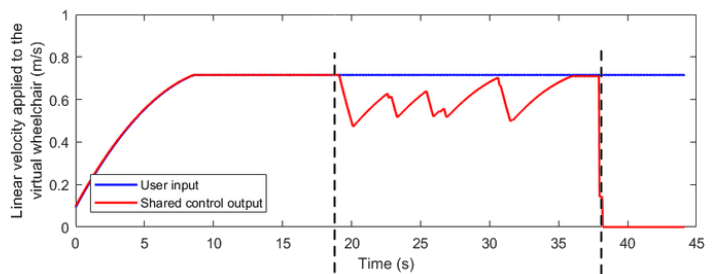

(b)

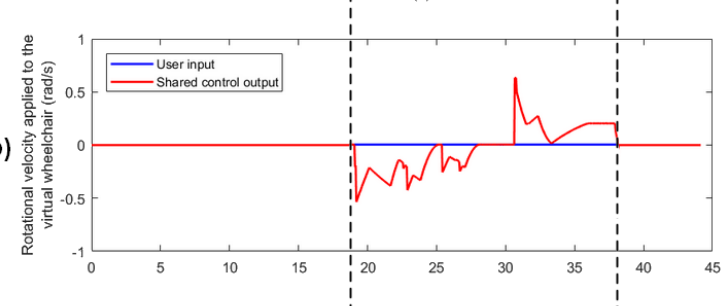

(c)

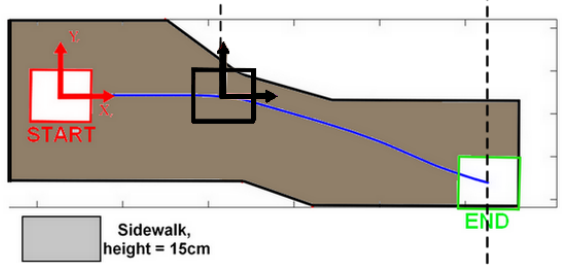

Fig. 9: Simulation results: (a) Forward velocity applied to the wheelchair (b) Rotational velocity applied to the wheelchair (c) Representation of the trajectory of the virtual wheelchair in the simulated environment (which we recorded for visualization purpose only). The rectangles represent the wheelchair at the beginning (red) and at the end (green) of the simulation.

solution has been developed and tested in simulation in the case of navigation in an environment with steep negative obstacles (e.g. curb, drop-off). Simulation results with different initial positions show our smart power wheelchair ability to avoid negative obstacles while respecting user intention (here driving forward). This is achieved by means of a smooth trajectory correction resulting from progressive regulation of the $2 \mathrm{DoF}$ wheelchair control inputs. The presented method necessitates an extrinsic calibration of the sensors as we use the sensor position information in our distance calculations. This consideration is easily respected when rigidly attaching the detection modules to the wheelchair frame thus ensuring a fixed position of the sensors. The findings obtained with the simulation framework encouraged us to equip a power wheelchair with such detection technology. Therefore, a standard M400 power wheelchair from permobil has been equiped with low-cost compliant Time-of-Flight (ToF) VL53L1X distance sensors from STMicroelectronics. The sensor-based shared control solution proposed in this paper is currently under tests. Videos showing wheelchair behavior in real environment conditions will be available soon at https://team.inria.fr/rainbow/fr/louise-devigne/. Finally, based on these results, simulations and tests will also be performed on the ability of the algorithm to provide relevant progressive assistance while negotiating a slope or a ramp. 


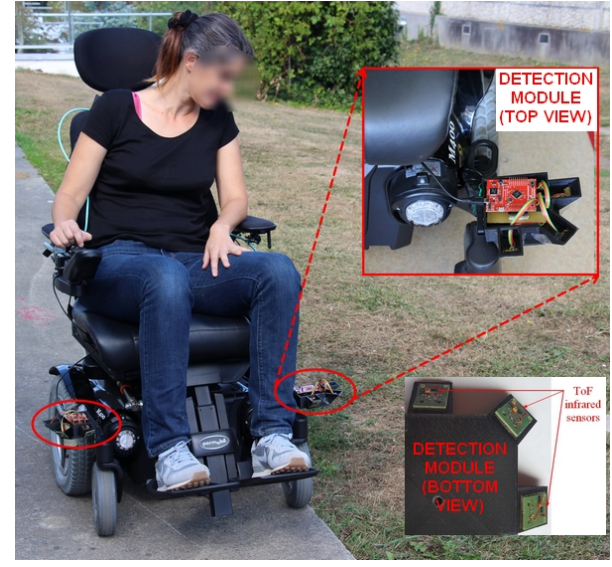

Fig. 10: On-going test on a real power wheelchair.

\section{CONCLUSION}

In this paper, we presented a shared-control solution for safe assisted power wheelchair navigation in an environment consisting of negative obstacles. This method regulates wheelchair control input values from constraints deduced from each sensor measured distance to the floor as well as local floor orientations. A gradual activation of the assistance allows the user to keep full wheelchair control when no obstacle is detected. When an obstacle such as a step is detected, a modular level of assistance allows progressive control input regulation. Therefore, in order to respect as much as possible user intention, user input is blended with a set of constraints deduced from the sensors. This provides an intuitive shared control scheme capable of assisting the user while needed without inducing a non-compliant trajectory. This approach coupled with the framework presented in [14] will allow a safe navigation indoors and outdoors.

The behavior of the system in simulation leads to validate our method. This proof-of-concept step is essential in order to technically validate the system before obtaining an ethical agreement for performing trials with non disabled volunteers as well as performing clinical trials with people with disabilities. The shared control model presented here is flexible, computationally inexpensive and independent of the range measurement sensor type. Moreover, this work showed the versatility of our method and encourages us to go further in our research process. In particular, future work will focus on improving the simulator physical engine in order to be able to test this solution of assisted power wheelchair navigation on a sidewalk to negotiate ramps.

\section{ACKNOWLEDGEMENT}

The authors would like to thank Éric Bazin from the IETR laboratory at INSA Rennes who was of great help during the development process.

This work has been funded by Inria associated team ISI4NAVE and by Institut des Neurosciences Cliniques de Rennes (INCR). This work is carried out as part of the INTERREG VA FMA ADAPT project "Assistive Devices for empowering disAbled People through robotic Technologies" http://adapt-project.com/index.php. The Interreg FCE Programme is a European Territorial Cooperation programme that aims to fund high quality cooperation projects in the Channel border region between France and England. The Programme is funded by the European Regional Development Fund (ERDF).

\section{REFERENCES}

[1] W.-Y. Chen, Y. Jang, J.-D. Wang, W.-N. Huang, C.-C. Chang, H.F. Mao, and Y.-H. Wang, "Wheelchair-related accidents: relationship with wheelchair-using behavior in active community wheelchair users," Archives of physical medicine and rehabilitation, vol. 92, no. 6, pp. 892-898, 2011.

[2] E. Baklouti, N. B. Amor, and M. Jallouli, "Autonomous wheelchair navigation with real time obstacle detection using $3 \mathrm{~d}$ sensor," $a u$ tomatika, vol. 57, no. 3, pp. 761-773, 2016.

[3] A. Kokosy, T. Floquet, G. Howells, H. Hu, M. Pepper, and C. Donzé, "SYSIASS - An Intelligent Powered Wheelchair," in Proc. of International Conference on Systems and Computer Science, 2012.

[4] O. Horn, "Smart wheelchairs: Past and current trends," in 2012 1st International Conference on Systems and Computer Science (ICSCS), Aug 2012, pp. 1-6.

[5] C. Ezeh, P. Trautman, L. Devigne, V. Bureau, M. Babel, and T. Carlson, "Probabilistic vs linear blending approaches to shared control for wheelchair driving," in IEEE Int. Conf. on Rehabilitation Robotics, ICORR'17, 2017.

[6] C. Löfqvist, C. Pettersson, S. Iwarsson, and A. Brandt, "Mobility and mobility-related participation outcomes of powered wheelchair and scooter interventions after 4-months and 1-year use," Disability and Rehabilitation: Assistive Technology, vol. 7, no. 3, pp. 211-218, 2012.

[7] K. Samuelsson and E. Wressle, "Powered wheelchairs and scooters for outdoor mobility: a pilot study on costs and benefits," Disability and Rehabilitation: AT, vol. 9, no. 4, pp. 330-334, 2014.

[8] J. M. Opalek, V. L. Graymire, and D. Redd, "Wheelchair falls: 5 years of data from a level i trauma center," Journal of trauma nursing, vol. 16, no. 2, pp. 98-102, 2009.

[9] B. Alexandra Korotchenko and P. Alex Mihailidis PhD, "Power mobility with collision avoidance for older adults: User, caregiver, and prescriber perspectives," Journal of rehabilitation research and development, vol. 50, no. 9, p. 1287, 2013.

[10] J. Larson and M. Trivedi, "Lidar based off-road negative obstacle detection and analysis," in Intelligent Transportation Systems (ITSC), 2011 International IEEE Conference on. IEEE, 2011, pp. 192-197.

[11] A. Murarka, M. Sridharan, and B. Kuipers, "Detecting obstacles and drop-offs using stereo and motion cues for safe local motion," in Intelligent Robots and Systems, 2008. IROS 2008. IEEE/RSJ International Conference on. IEEE, 2008, pp. 702-708.

[12] R. Simpson, E. LoPresti, S. Hayashi, I. Nourbakhsh, and D. Miller, "The smart wheelchair component system," Journal of Rehabilitation Research and Development, vol. 41, no. 3B, p. 429, 2004.

[13] J. M. Coughlan and H. Shen, "Terrain analysis for blind wheelchair users: Computer vision algorithms for finding curbs and other negative obstacles." in CVHI, 2007.

[14] L. Devigne, V. K. Narayanan, F. Pasteau, and M. Babel, "Low complex sensor-based shared control for power wheelchair navigation," in Intelligent Robots and Systems (IROS), 2016 IEEE/RSJ International Conference on. IEEE, 2016, pp. 5434-5439.

[15] O. Kermorgant and F. Chaumette, "Combining ibvs and pbvs to ensure the visibility constraint." in IROS, 2011, pp. 2849-2854.

[16] F. Pasteau, A. Krupa, and M. Babel, "Vision-based assistance for wheelchair navigation along corridors," in Robotics and Automation (ICRA), 2014 IEEE International Conference on. IEEE, 2014, pp. 4430-4435.

[17] B. Espiau, F. Chaumette, and P. Rives, "A new approach to visual servoing in robotics," ieee Transactions on Robotics and Automation, vol. 8, no. 3, pp. 313-326, 1992.

[18] L. Devigne, M. Babel, F. Nouviale, V. Narayanan, F. Pasteau, and P. Gallien, "Design of an immersive simulator for assisted power wheelchair driving," in IEEE Int. Conf. on Rehabilitation Robotics, ICORR'17, 2017. 